\title{
COVID-19 and Radiologist: Image Wisely
}

\begin{abstract}
On March 11, 2020, coronavirus disease-2019 (COVID-19) was announced as a pandemic by the World Health Organization (WHO). The WHO urged for international action and co-operation in the following key areas: adequate preparation and readiness; detection, protection, and treatment; reducing transmission; and innovation and learning. As this disease has caused a significant impact on our lives, the health-care workers are the worst hit, both mentally and physically. In such hours of crisis where this pandemic is spreading rapidly, our role is to prudently use the health resources and relieve any extra burden on the health-care workers. This article reviews the role of imaging in the management of COVID-19 and also discusses the recommendations and guidelines for judicious use of imaging.
\end{abstract}

Keywords: Chest radiograph, computed tomography, coronavirus, COVID-19, imaging, nCOV, point-of-care ultrasound, radiology

\section{Introduction}

On March 11, 2020, COVID-19 was announced as a pandemic by the World Health Organization (WHO). The WHO urged for international action and co-operation in the following key areas: Adequate preparation and readiness; detection, protection, and treatment; reducing transmission; and innovation and learning. As of April 9, 2020, there are approximately 1,395,136 reported cases and 81,580 deaths across the world [Figure 1a and b]. Around 212 countries, areas or territories have reported COVID cases. $^{[1-3]}$ The worst-hit countries include Italy, Spain, the USA, and China. Currently, India has been also working on strategies to combat disease spread using social distancing and imposed quarantine. India has declared a nationwide lockdown until April 30, 2020. Considering this disease has caused a significant impact on all our lives, the health-care workers are the worst hit, both mentally and physically. In hours of crisis such as these with a rapidly spreading pandemic, our role is to ensure a judicious use of health resources and relieve any extra burden on the health-care workers. $^{[4-6]}$ This article reviews the role of imaging in the management of COVID-19; also discusses the recommendations and guidelines for prudent use of imaging.

This is an open access journal, and articles are distributed under the terms of the Creative Commons Attribution-NonCommercial-ShareAlike 4.0 License, which allows others to remix, tweak, and build upon the work non-commercially, as long as appropriate credit is given and the new creations are licensed under the identical terms.

For reprints contact: WKHLRPMedknow_reprints@wolterskluwer.com

\section{Coronavirus Disease-2019 Diagnosis}

Coronavirus disease-2019 (COVID-19) is an infectious disease caused by severe acute respiratory syndrome coronavirus 2 (SARS-CoV-2), previously called as 2019 novel coronavirus (2019-nCoV). The disease spreads via droplet transmission with a median incubation period of 5.1 days and $97.5 \%$ becoming symptomatic within 11.5 days (CI, 8.2-15.6 days) of getting infected. This extends to the $99^{\text {th }}$ percentile results in almost all cases developing symptoms in 14 days after exposure. The R0 (basic reproduction number) is estimated to be between 2.2 and 3.28; this means that each infected individual, on average, causes 2-3 new infections. ${ }^{[7,8]}$ The common presenting symptoms include fever $(85 \%-90 \%)$, cough $(65 \%-70 \%)$, fatigue and myalgia $(35 \%-40 \%)$, sputum production $(30 \%-35 \%)$, and shortness of breath $(15 \%-20 \%)$. The less common symptoms include arthralgia $(10 \%-15 \%)$, headaches $(10 \%-15 \%)$, sore throat $(10 \%-15 \%)$, chills $(10 \%-12 \%), \quad$ and pleuritic pain. The overall mortality rate ranges from $2.3 \%$ to $2.9 \%$. The disease severity has been classified into three grades: mild grade - no symptoms, mild coughing, and fever; severe grade - dyspnea, hypoxia, or $>50 \%$ lung involvement on imaging; and critical grade - respiratory failure, shock, multi-organ failure. Overall, approximately

\footnotetext{
How to cite this article: Mahajan A, Sharma $P$. COVID-19 and radiologist: Image wisely. Indian J Med Paediatr Oncol 2020;41:121-6.
}

\section{Abhishek Mahajan', Prerit Sharma ${ }^{2}$}

${ }^{1}$ Department of Radiodiagnosis and Imaging, Tata Memorial Centre, Tata Memorial Hospital, Mumbai, Maharashtra, India, ${ }^{2}$ Department of Radiodiagnosis and Imaging, Sharma Diagnostics, Wardha, Maharashtra, India

Submitted: 09-Apr-2020 Revised: 16-Apr-2020 Accepted: 17-Apr-2020 Published: 30-May-2020

Address for correspondence: Dr. Abhishek Mahajan, Department of Radiodiagnosis and Imaging, Tata Memorial Centre, Tata Memorial Hospital, Mumbai - 400 012, Maharashtra, India.

E-mail: drabhishek.mahajan@ yahoo.in 


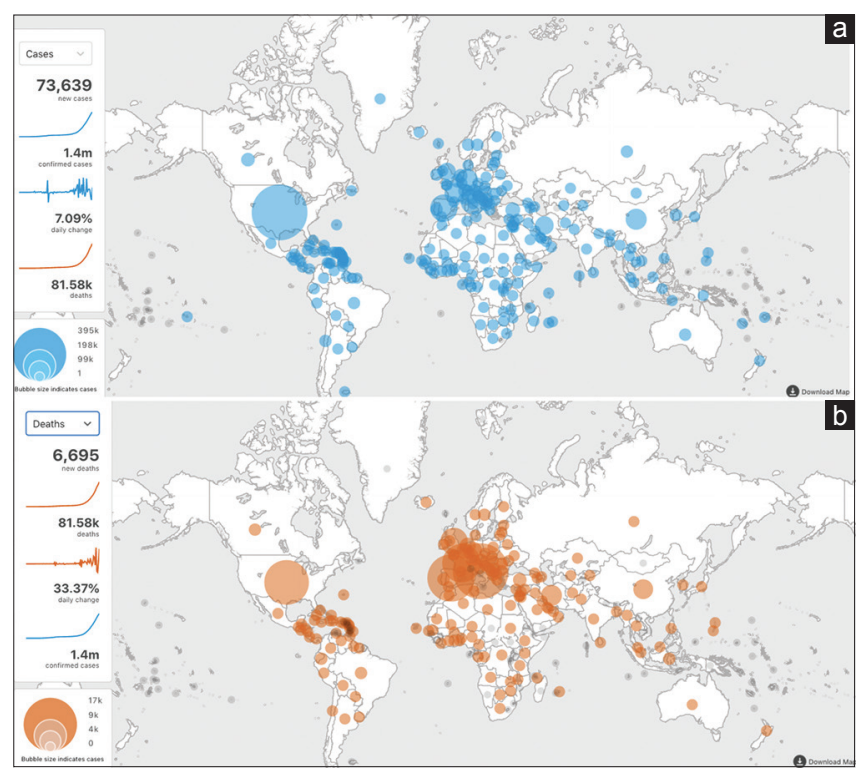

Figure 1: ( $a$ and b) COVID-19 cases and deaths across the globe (source: ref 3 )

$1 \%$ of cases are asymptomatic and approximately $80 \%$ are classified as "mild." In the pediatric population, the disease is seen to have a shorter incubation period with a milder course and lower mortality. Accordingly to a study, approximately $6 \%$ of the total cases needed invasive or noninvasive intensive care unit (ICU). This group comprised predominantly elderly patients and at-risk population (with comorbid illnesses such as uncontrolled diabetes, recent myocardial infarction, and uncontrolled hypertension). ${ }^{[9,10]}$ The commonly reported indications for ICU admission included acute respiratory distress syndrome (ARDS) (approximately 23\%), acute cardiac injury, super-added infections, sepsis, acute renal failure, and rarely, cytokine storm syndrome.

The real-time reverse transcriptase-polymerase chain reaction (rRT-PCR) is a very specific diagnostic test but has a lower sensitivity of $65 \%-95 \%$, which implies that there are chances of a false-negative test result even when the patient is infected. It is also time consuming and takes at least $24 \mathrm{~h}$ for the results. The other laboratory findings include a reduced lymphocyte count and an increased C-reactive protein, prothrombin time, and lactate dehydrogenase. ${ }^{[11,12]}$

\section{Coronavirus Disease-2019 and Imaging}

As this pandemic becomes a global threat, the health-care system seems to be in the utmost pressure. It seems worthwhile to make judicious use of imaging in the management of COVID-19. Imaging in COVID-19 can be discussed into four subheadings: different imaging modalities, indications, imaging findings and reporting, and personal protective equipment (PPE) and safe imaging.

\section{Imaging Modalities}

It is prudent to avoid unnecessary imaging and commotion of suspected or positive cases to and from the radiology department. The frequency of imaging should be patient specific and rather not be pre-defined. The most economical and recommended imaging modality is chest radiography (CXR). A portable X-ray system may be deployed to a COVID-19 ward or isolation ward which is used exclusively for imaging COVID-suspected or COVID-confirmed patients. Although the sensitivity of CXR is low $(<70 \%)$ as compared to computed tomography (CT) of the thorax, CT is still not recommended for diagnosing COVID-19. The third imaging modality is point-of-care ultrasound (POCUS). POCUS has shown a significant impact on ICU patients not only in terms of its diagnostic ability but also in limiting the rate of cross-contamination. Lung ultrasound is a safe, rapid, and a promising tool in diagnosing this contagious viral disease, especially in the ICU setting. ${ }^{[13-15]}$

\section{Clinical Indications}

The indications for imaging can be divided into four aspects and are summarized in Figure 2: ${ }^{[13-16]}$

1. Screening: Imaging has no role in screening of COVID-19 and is strongly discouraged

2. COVID-suspected individuals: Imaging is not recommended for confirming the diagnosis of COVID-19. However, in individuals with high clinical suspicion, strong contact history, and negative RT-PCR results, CT imaging should be used to further investigate the classical COVID-19 findings which can serve as a surrogate marker and emphasize the need for a repeat RT-PCR. In situations with delayed RT-PCR results; CXR may play a significant role in patients with fever, hypoxia (spo2 <or equal to 94\%), and tachypnea (respiratory rate >or equal to 20 )

3. COVID-confirmed individuals: In patients with RT-PCR-confirmed COVID-19, imaging is not routinely recommended. Normal imaging on both CXR and CT of the thorax does not rule out COVID-19. Patients showing a milder form of disease do not require imaging. However, with moderate and severe disease, the baseline CXR may be performed to evaluate the evolution of the disease. Follow-up imaging in these scenarios should be used cautiously and it may vary from case to case. Depending on the availability, either POCUS or CT of the thorax might be necessary for severe cases with a significant clinical deterioration. These imaging tools can help to rule out other causes of sudden deterioration other than ARDS. They are also helpful in diagnosing secondary complications and superadded infections. CT of the brain may be indicated in patients with neurological deterioration and suspected acute necrotizing encephalopathy

4. COVID-incidental: More and more individuals are presenting with abdominal symptoms such as intestinal obstruction, gastrointestinal (GI) bleed, and GI inflammation. In these atypical scenarios, if indicated, 


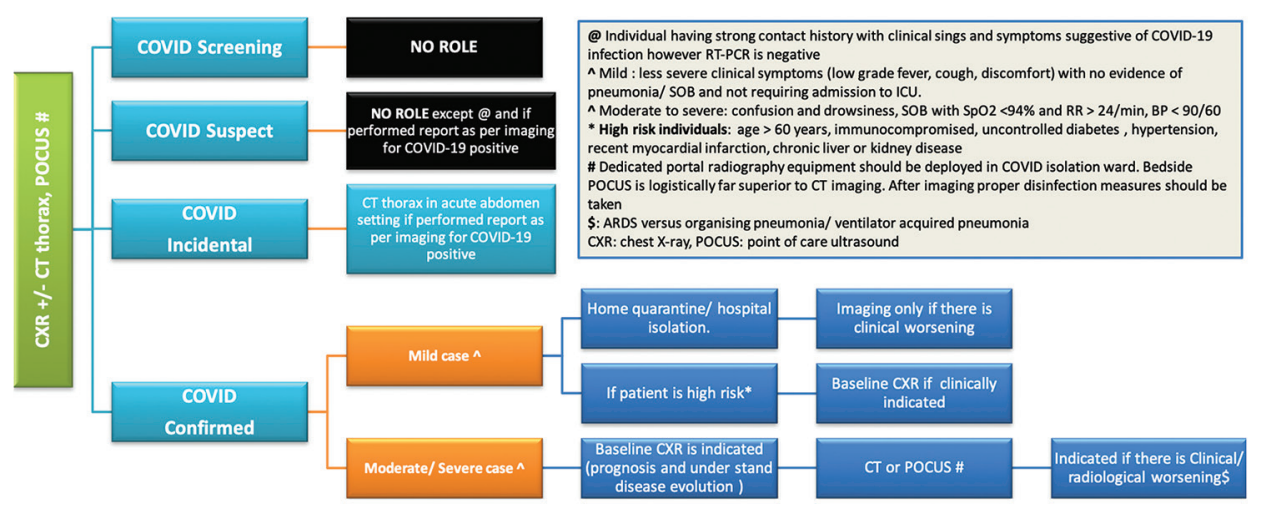

Figure 2: Guidelines and recommendations for imaging in COVID-19. Adapted from Ahuja et al. ${ }^{[36]}$

a CT of the thorax must be included and the CT of the thorax findings should be reported as that for COVID suspect.

\section{Imaging Findings and Reporting of Coronavirus Disease-2019}

\section{Chest X-ray}

Approximately $70 \%$ of the patients requiring hospitalization have initial baseline CXR abnormalities and approximately $90 \%$ have detectable CXR abnormalities during the hospital stay. CXR tends to be display positive findings during the $2^{\text {nd }}$ week of SARS-CoV-2 infection and findings are most prevalent approximately 10-12 days postinfection. The most frequent finding is airspace shadows, typically described as consolidation and less commonly ground-glass opacities (GGO). They are bilateral, peripheral, and predominantly in lower zones. Other rare CXR findings include pleural effusion, pneumothorax, and interstitial pattern. Findings of ARDS may be seen in severe cases with dropping Spo2. ${ }^{[16,17]}$

\section{Computed tomography of the thorax}

Plain CT of the thorax is adequate and appropriate for imaging COVID-19 patients. The primary findings on CT in adult patients with COVID-19 are GGOs (88\%) that are typically bilateral $(87 \%)$ and subpleural and peripheral (76\%); crazy paving appearance (GGOs and inter/intralobular septal thickening); air space consolidation (32\%); bronchovascular thickening in the lesion; and traction bronchiectasis. Other rare CT findings include pleural effusion, pericardial effusion, lymphadenopathy, halo sign, pleural thickening, and pneumothorax. In one study, approximately $50 \%$ of the asymptomatic cases had positive chest CT findings.

In patients with frank hemoptysis, contrast CT imaging is indicated to rule out the cause of hemoptysis. However, it is recommended to always do a plain $\mathrm{CT}$ prior to contrast $\mathrm{CT}$ as the contrast may impact the evaluation of GGOs.

CT has a limited role in confirming the diagnosis of SARS-CoV-2 infection (normal CT does not rule out
COVID-19!!). The overall diagnostic accuracy of CT in terms of sensitivity, specificity, PPV, and negative predictive value (NPV) is $60 \%-98 \%, 25 \%-53 \%, 92 \%$, and $42 \%$, respectively. The low NPV makes CT not suitable for screening COVID-19 suspects. ${ }^{[18-21]}$ Some authors have anticipated that the incidence of coexisting infections could be as high as $20 \%$, and these patients may demonstrate mixed (typical and atypical) CT findings. ${ }^{[16]}$

CT findings show temporal changes during the course of disease with focal GGOs and consolidation in the ultra-early stage (1-2 weeks after exposure); GGO combined with interlobular septal thickening in the early stage (day 1-3 after clinical manifestation); extensive consolidative patches with air bronchograms are the common findings in the rapid progression stage (days 3-7 of symptomatic presentation); and during the consolidative stage ( $2^{\text {nd }}$ week since clinical presentation), the lesions start to involute and gradually regress in size and number. The incidence of GGO and consolidation varies with age, and the incidence of GGOs was higher $(>70 \%)$ in patients younger than 50 years. However, in patients older than 50 years, consolidation and GGO had equal incidence. ${ }^{[22-24]}$ Known lung carcinoma does not seem to have any influence over the course of COVID-19. ${ }^{[25]}$

\section{Point-of-Care Ultrasound}

Bedside lung ultrasonography (USG) is a practical alternative to $\mathrm{CT}$ imaging for critical patients, especially in the ICU. The findings on ultrasound USG are predominantly posterobasal and bilateral; common findings include multiple B-lines that represent thickened subpleural interlobular septae, subpleural consolidative patches, irregular thickened pleura, and pleural effusion. In severe progressive disease, alveolar consolidation is a common finding which is seen as a tissue-like appearance with dynamic and static air bronchograms. ${ }^{[26-28]}$

\section{Reporting of Imaging Findings}

In scenarios where CXR or/and CT thorax are performed for either COVID-suspected or COVID-confirmed patients, 
the reports should include a CT severity score. The severity of lung involvement on imaging correlates with disease severity. Both the lungs can be divided into three zones on each side: Zone 1 - apex to the carina, Zone 2 - carina to the inferior pulmonary vein, and Zone 3 - below the inferior pulmonary vein. Each zone can be assigned a visual score of 0-4 (no abnormality - 0, up to $25 \%$ involvement -1 , up to $50 \%$ involvement -2 , up to $75 \%$ involvement -3 , and $>75 \%$ involvement score -4$)$. The severity grades include mild, moderate and severe.

The final impression should be categorized as one of the following: typical for COVID-19, indeterminate for COVID-19, atypical for COVID-19 (look for other causes), and negative for pneumonia. ${ }^{[16]}$ The typical category comprises few characteristic findings in the following patterns - peripheral bilateral GGOs with/without consolidation or visible intralobular lines ("crazy-paving") or multifocal GGO of rounded morphology with or without consolidation or visible intralobular lines ("crazy paving") or reverse halo sign or other findings of organizing pneumonia (later stage of the disease) [Figure 3]. These findings can also be seen in the COVID-19 and can also be seen in some other conditions such as viral pneumonia of other causes (especially in influenza) and acute lung injury patters such as organizing pneumonia [Figure 4a] secondary to drug toxicities/ graft versus host disease [Figure 4b] or connective tissue disorders or idiopathic [Figure $4 \mathrm{c}] .^{[16]}$

The indeterminate appearance on CT of the thorax is usually the absence of typical features with other findings such as multifocal, diffuse, perihilar, or unilateral GGO with/without consolidation lacking a specific distribution and is nonrounded or nonperipheral. Few very small

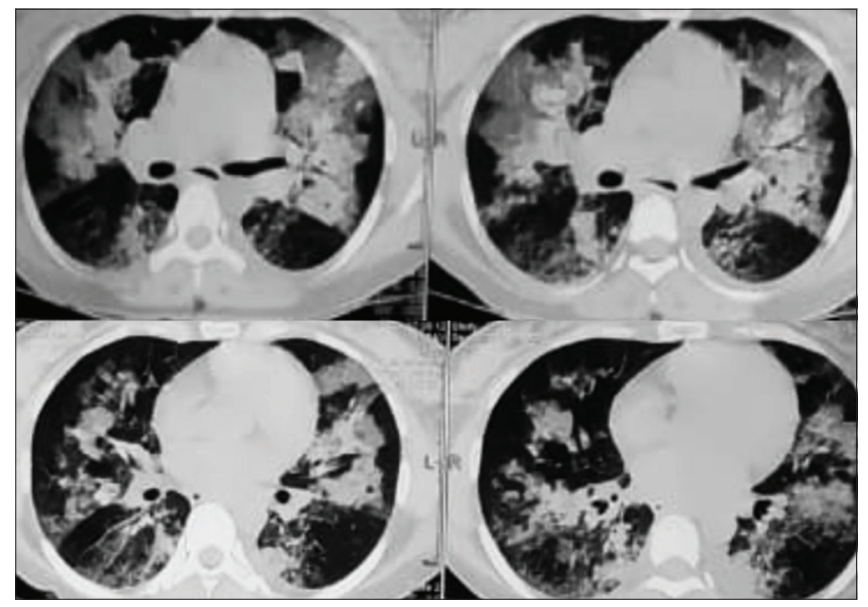

Figure 3: A 33-year-old pregnant female underwent a lower (uterine) segment cesarean section, and on day 2, the patient developed fever and cough with a sudden onset of breathlessness; the computed tomography of the thorax was performed to rule of pulmonary thromboembolism. Computed tomography showed peripheral patchy areas of consolidation with surroundings ground-glass opacities and subpleural lines and crazy paving typical for COVID-19. The patient was shifted to the ICU and was on ventilator
GGOs with a nonrounded and nonperipheral distribution can also be considered as an indeterminate appearance. These features can also be seen in other conditions such as acute hypersensitivity pneumonitis, pneumocystis infections [Figure 4d], and diffuse alveolar hemorrhage. They are not specific enough to be to make a confident diagnosis..$^{[16]}$

The atypical appearance usually should be considered when there is an absence of both typical and indeterminate features but instead show isolated lobar or segmental consolidation with GGO (as seen in bacterial pneumonia) or presence of discrete small nodules predominantly centrilobular giving a "tree-in-bud" appearance (such as in community-acquired or aspiration pneumonia) or lung cavitation (seen in necrotizing pneumonia) or smooth interlobular septal thickening with pleural effusion. In such cases, alternative diagnoses such as bacterial pneumonia, necrotizing pneumonia, and community-acquired or aspiration pneumonia should be considered. ${ }^{[16]}$

The scan can be reported as negative for pneumonia if the CT scan demonstrates no features suggestive of pneumonia. ${ }^{[16]}$

\section{Personal Protective Equipment and Safe-imaging ${ }^{[29-35]}$}

It is important to follow PPE guidelines to limit cross-contamination and nosocomial spread. PPE may include masks (N95 respirator or surgical masks but not a cloth mask), gloves, eye protection goggles, gowns, face shields, hoods, and shoe covers. If logistically feasible, dedicated COVID-CT equipment should be made available. If logistically feasible, dedicated equipment is recommended for the imaging of only COVID-19-positive

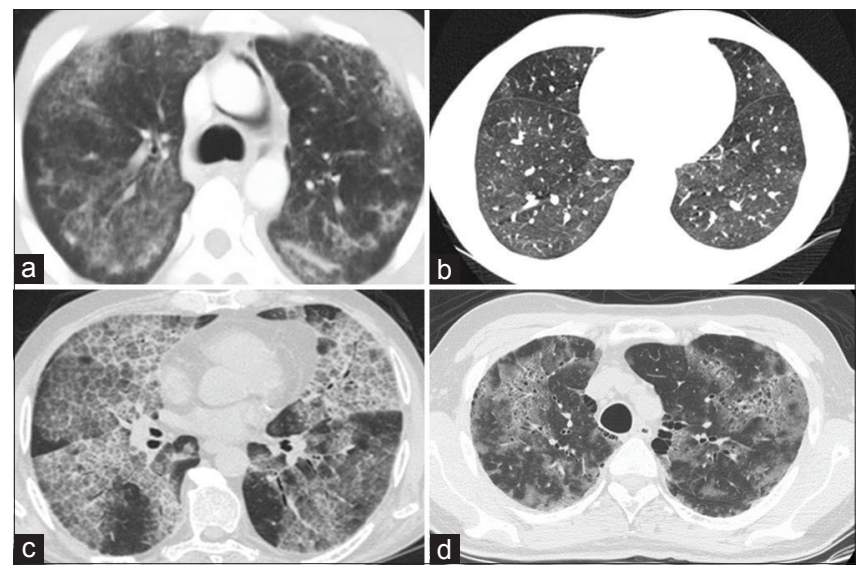

Figure 4: (a-d) COVID-19 mimics. Patchy consolidation with a predominantly subpleural and peribronchial distribution as seen in this case of cryptogenic organizing pneumonia (a). Scattered nodules, areas of ground-glass opacities, and air trapping as seen in a case of bronchiolitis obliterans due to graft versus host disease (b). Extensive crazy paving and reticular pulmonary fibrosis seen in a case of secondary pulmonary alveolar proteinosis (c). Reticular opacities with crazy paving and mosaic patterns of ground-glass opacities with consolidation in a case of pneumocystis pneumonia (d) 
patients in the allotted ward, as in that case, equipment sanitization will be not required after every procedure. No patient coming to the department for COVID-19-related imaging should be without a well-placed face mask. Manual handling of referral forms for imaging should be avoided to limit cross-contamination. Adequate infection control measures should be undertaken without any delay once the imaging procedure is performed. The CT room should be cleaned with a $1 \%$ sodium hypochlorite solution, and the areas of contact should be cleaned with alcohol-based disinfectants. The CT room should be vacated empty with strictly no permission for any patients/ health-care worker to enter for at least $1 \mathrm{~h}$ with a passage for fresh air to thoroughly ventilate the room. Follow institutional reuse policies for masks. Strict standard operating procedures (SOPs) and guidelines should be laid down (CDC, WHO, and RSNA) for all the imaging modalities and imaging staff.

\section{Conclusion}

We as health-care workers have the onus to make judicious use of imaging resources and follow strict SOPs to combat this pandemic. Imaging has no role in screening of suspected COVID-19 cases with exceptions described above. In COVID-19 cases, the use of imaging is based on the clinical status of patients preferably reserved for moderate-to-severe cases and in patients with sudden clinical deterioration. POCUS or bedside ultrasound is a practical alternative to $\mathrm{CT}$ imaging for critical patients in the ICU. PPE and sanitization procedures must be followed to limit nosocomial spread.

\section{Declaration of patient consent}

The authors certify that they have obtained all appropriate patient consent forms. In the form the patient(s) has/have given his/her/their consent for his/her/their images and other clinical information to be reported in the journal. The patients understand that their names and initials will not be published and due efforts will be made to conceal their identity, but anonymity cannot be guaranteed.

\section{Acknowledgments}

The authors would like to thank Indian Radiological and Imaging Association and Indian College of Radiology and Imaging (ICRI), Imaging recommendation by ICRI for COVID-19 by Dr. Ashu Seth Bhalla, Dr. Vimal Raj, Dr. Abhishek Mahajan, Dr. Leena R V, Dr. Lalendra Upreti Dr. Priyanka Naranje, Dr. Gh Mohammad Wani, Dr. Anant Mohan, Dr. Mandeep Garg, Dr. Anindita Sinha, Dr. Aparna Irodi, and Dr. Smita Manchanda.

\section{Financial support and sponsorship}

Nil.

\section{Conflicts of interest}

There are no conflicts of interest.

\section{References}

1. Available from: https://www.who.int/emergencies/diseases/ novel-coronavirus-2019. [Last accessed on 2020 Apr 09].

2. WHO Director-General's Opening Remarks at the Media Briefing on COVID-19-11 March 2020; 2020. Available from: https://www.who.int/dg/speeches/detail/who-director-generals-opening-remarks-at-the-media-briefing-on-covid-19---11march-2020. [Last accessed on 2020 Apr 09].

3. Available from: https://who.sprinklr.com/. [Last accessed on 2020 Apr 09].

4. Velavan TP, Meyer CG. The COVID-19 epidemic. Trop Med Int Health 2020;25:278-80.

5. Heymann DL, Shindo N. COVID-19: What is next for public health?. Lancet 2020;395:542-5.

6. Mandal S, Bhatnagar $\mathrm{T}$, Arinaminpathy $\mathrm{N}$, Agarwal A, Chowdhury A, Murhekar M, et al. Prudent public health intervention strategies to control the coronavirus disease 2019 transmission in India: A mathematical model-based approach. Indian J Med Res 2020. doi: 10.4103/ijmr.IJMR_504_20. [Epub ahead of print].

7. Zhu N, Zhang D, Wang W, Li X, Yang B, Song J, et al. A novel coronavirus from patients with pneumonia in China, 2019. N Engl J Med 2020;382:727-33.

8. Chen N, Zhou M, Dong X, Qu J, Gong F, Han Y, et al. Epidemiological and clinical characteristics of 99 cases of 2019 novel coronavirus pneumonia in Wuhan, China: A descriptive study. Lancet 2020;395:507-13.

9. Guan WJ, Ni ZY, Hu Y, Liang WH, Ou CQ, He JX, et al. China Medical Treatment Expert Group for Covid-19 (2020) Clinical characteristics of coronavirus disease 2019 in China. N Engl J Med 2020. doi: 10.1056/NEJMoa2002032. [Epub ahead of print].

10. Wu Z, McGoogan JM. Characteristics of and important lessons from the coronavirus disease 2019 (COVID-19) outbreak in China: Summary of a report of 72314 cases from the Chinese Center for Disease Control and Prevention. JAMA 2020. doi: 10.1001/jama.2020.2648. [Epub ahead of print].

11. Tsang J, Bajpai J. Novel coronavirus infection - Knowns and unknowns with particular reference to oncology - Combating against COVID19 with "COVID." Indian J Med Paediatr Oncol [Epub ahead of print]. Available from: http://www.ijmpo.org/ preprintarticle.asp?id=281591. [cited $2020 \mathrm{Apr}$ 27].

12. Lan L, Xu D, Ye G, Xia C, Wang S, Li Y, et al. Positive RT-PCR test results in patients recovered from COVID-19. JAMA 2020. doi: 10.1001/jama.2020.2783. [Epub ahead of print].

13. American College of Radiology. COVID-19 Radiology-Specific Resources. Available from: https://www.acr.org/ Clinical-Resources/COVID-19-Radiology-Resources. [Last accessed on 2020 Apr 09].

14. Rodrigues JC, Hare SS, Edey A, Devaraj A, Jacob J, Johnstone A, et al. An update on COVID-19 for the radiologist-A British society of Thoracic Imaging statement. Clin Radiol 2020;75:323-5.

15. Lee EY, Ng MY, Khong PL. COVID-19 pneumonia: what has CT taught us? Lancet Infect Dis 2020;20:384-5.

16. Simpson S, Kay FU, Abbara S, Bhalla S, Chung JH, Chung M, et al. Radiological society of North America expert consensus statement on reporting chest CT findings related to COVID-19. Endorsed by the Society of Thoracic Radiology, the American College of Radiology, and RSNA. Radiol Cardiothorac Imaging 2020;2:e200152.

17. Rodriguez-Morales AJ, Cardona-Ospina JA, Gutiérrez-Ocampo E, Villamizar-Peña R, Holguin-Rivera Y, Escalera-Antezana JP, et al. Clinical, laboratory and imaging features of COVID-19: 
A systematic review and meta-analysis. Travel Med Infect Dis 2020 Mar 13:101623. doi: 10.1016/j.tmaid.2020.101623. [Epub ahead of print].

18. Pan F, Ye T, Sun P, Gui S, Liang B, Li L, et al. Time course of lung changes on chest CT during recovery from 2019 novel coronavirus (COVID-19) pneumonia. Radiology 2020. Published Online:Feb 13 2020. https://doi.org/10.1148/radiol.2020200370.

19. Pan $\mathrm{Y}$, Guan $\mathrm{H}$, Zhou $\mathrm{S}$, Wang $\mathrm{Y}, \mathrm{Li} \mathrm{Q}, \mathrm{Zhu} \mathrm{T}$, et al. Initial $\mathrm{CT}$ findings and temporal changes in patients with the novel coronavirus pneumonia (2019-nCoV): A study of 63 patients in Wuhan, China. Eur Radiol 2020. doi: 10.1007/s00330-02006731-x. [Epub ahead of print].

20. Kanne JP, Little BP, Chung JH, Elicker BM, Ketai LH. Essentials for radiologists on COVID-19: An update-radiology scientific expert panel. Radiology 2020 Feb 27:200527. doi: 10.1148/ radiol.2020200527. [Epub ahead of print].

21. Zhao W, Zhong Z, Xie X, Yu Q, Liu J. Relation between chest $\mathrm{CT}$ findings and clinical conditions of coronavirus disease (COVID-19) pneumonia: A multicenter study. Am J Roentgenol 2020;214:1072-7. doi: 10.2214/AJR.20.22976. Epub 2020 Mar 3.

22. Salehi S, Abedi A, Balakrishnan S, Gholamrezanezhad A. Coronavirus disease 2019 (COVID-19): A systematic review of imaging findings in 919 patients. Am J Roentgenol 2020 Mar 14:1-7. doi: 10.2214/AJR.20.23034. [Epub ahead of print].

23. Jin YH, Cai L, Cheng ZS, Cheng H, Deng T, Fan YP, et al. A rapid advice guideline for the diagnosis and treatment of 2019 novel coronavirus (2019-nCoV) infected pneumonia (standard version). Military Med Res 2020;7:4.

24. Song F, Shi N, Shan F, Zhang Z, Shen J, Lu H, et al. Emerging 2019 novel coronavirus (2019-nCoV) pneumonia. Radiology 2020;295:210-7. doi: 10.1148/radiol.2020200274. Epub 2020 Feb 6.

25. Qu J, Yang R, Song L, Kamel IR. Atypical lung feature on chest $\mathrm{CT}$ in a lung adenocarcinoma cancer patient infected with COVID-19. Ann Oncol 2020 Mar 9. pii: S0923-7534(20)360804. doi: 10.1016/j.annonc.2020.03.001. [Epub ahead of print].

26. Peng QY, Wang XT, Zhang LN; Chinese Critical Care Ultrasound
Study Group. Findings of lung ultrasonography of novel corona virus pneumonia during the 2019-2020 epidemic. Intensive Care Med 2020 Mar 12. doi: 10.1007/s00134-020-05996-6. [Epub ahead of print].

27. Sripathi S, Mahajan A. Comparative study evaluating the role of color Doppler sonography and computed tomography in predicting chest wall invasion by lung tumors. J Ultrasound Med 2013;32:1539-46.

28. Buonsenso D, Pata D, Chiaretti A. COVID-19 outbreak: Less stethoscope, more ultrasound. Lancet Respir Med 2020. pii: S2213-2600 (20) 30120-X.

29. Centres for Disease Control and Prevention. Coronavirus 2019 (COVID-19)-Resources for Clinics and Healthcare Facilities. Available from: https:/www.cdc.gov/coronavirus/2019-ncov/hcp/ index.html?CDC_AA_refVal=https $\% 3 \mathrm{~A} \% 2 \mathrm{~F} \% 2 \mathrm{Fwww} . c d c . g o v \%$ 2 Fcoronavirus $\% 2$ F 2019 -ncov $\% 2$ Fhealthcare-facilities $\% 2$ Findex. html. [Last accessed on 2020 Apr 09].

30. Kooraki S, Hosseiny M, Myers L, Gholamrezanezhad A. Coronavirus (COVID-19) outbreak: What the department of radiology should know. J Am Coll Radiol 2020;17:447-51.

31. Nakajima $K$, Kato $H$, Yamashiro $T$, Izumi $T$, Takeuchi $I$, Nakajima H, et al. COVID-19 pneumonia: infection control protocol inside computed tomography suites. Jpn J Radiol 2020 Mar 17. doi: 10.1007/s11604-020-00948-y. [Epub ahead of print].

32. Available from: https://icmr.nic.in/content/covid-19. [Last accessed on 2020 Apr 09].

33. Available from: https://www.covid19india.org/. [Last accessed on 2020 Apr 09].

34. Available from: https://www.mohfw.gov.in/. [Last accessed on 2020 Apr 09].

35. Bhalla AS, Raj V, Mahajan A, Leena RV, Upreti L, Naranje P, et al. Imaging recommendation by Indian Radiological and Imaging Association (IRIA) and Indian College of Radiology and Imaging (ICRI) for COVID-19. [In press].

36. Ahuja A, Mahajan A. Imaging and COVID-19: Preparing the radiologist for the pandemic. Cancer Res Stat Treat 2020;3 Suppl S1:80-5. 